Supporting information for

\title{
Mesoporous CoP Nanowire Arrays for Hydrogen
}

\section{Evolution}

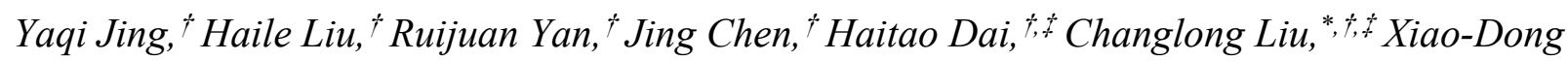

Zhang ${ }^{*}, t, t$

$\dagger^{\dagger}$ Department of Physics, School of Science, Tianjin University, Tianjin 300350, PR, China

$\$$ Tianjin Key Laboratory of Low Dimensional Materials Physics and Preparation Technology, Institute of Advanced Materials Physics, School of Science, Tianjin 300350, PR, China

* Corresponding authors.

E-mail addresses: xiaodongzhang@tju.edu.cn (X. Zhang), liuchanglong@tju.edu.cn (C. Liu). 
Supplementary Figures

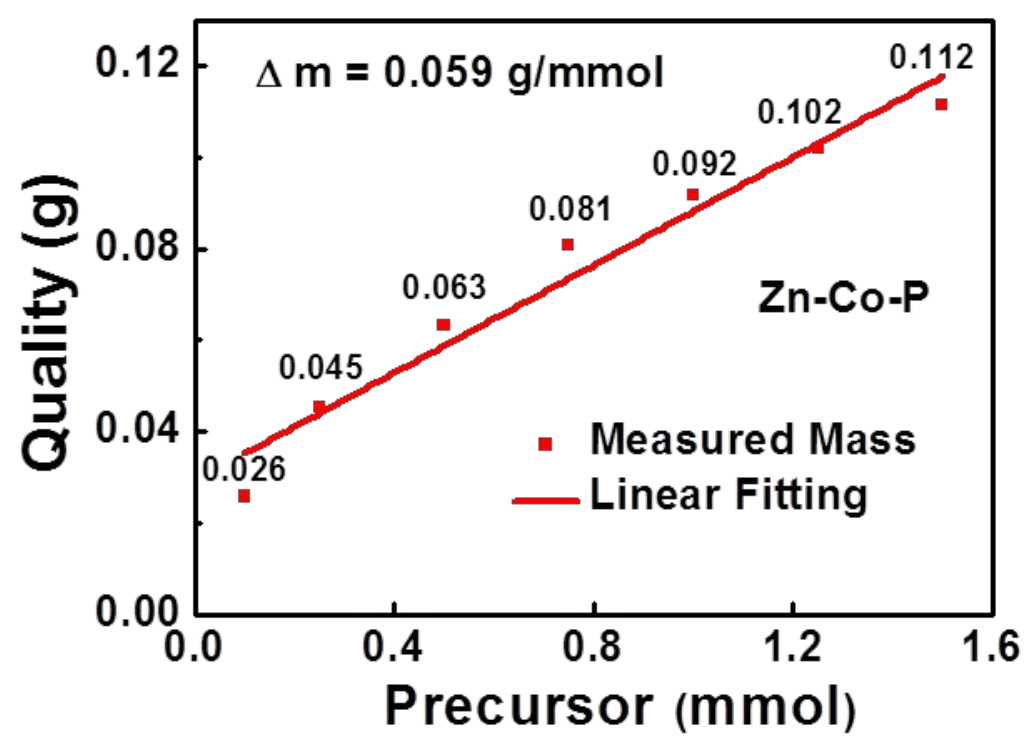

Figure S1. The almost-linear relationship between the amount of precursor $\left(\mathrm{Co}^{2+}, \mathrm{Zn}^{2+}\right)$ and the loading quantity of $\mathrm{Zn}-\mathrm{Co}-\mathrm{P}$. 


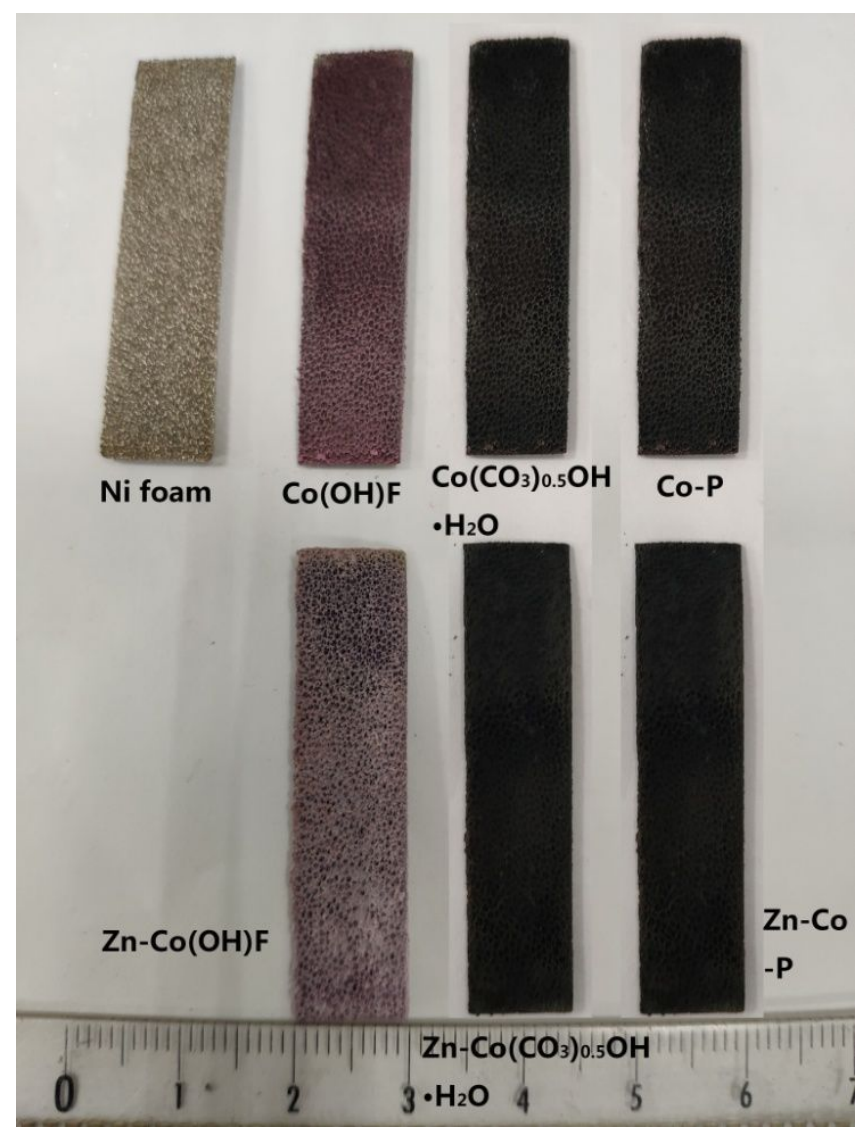

Figure S2. Optical photograph of the precursors in different stages of synthesis. After the hydrothermal step, the color of $\mathrm{Ni}$ foam turned from silvery white to red and further changed to black after air annealing and phosphorization. 

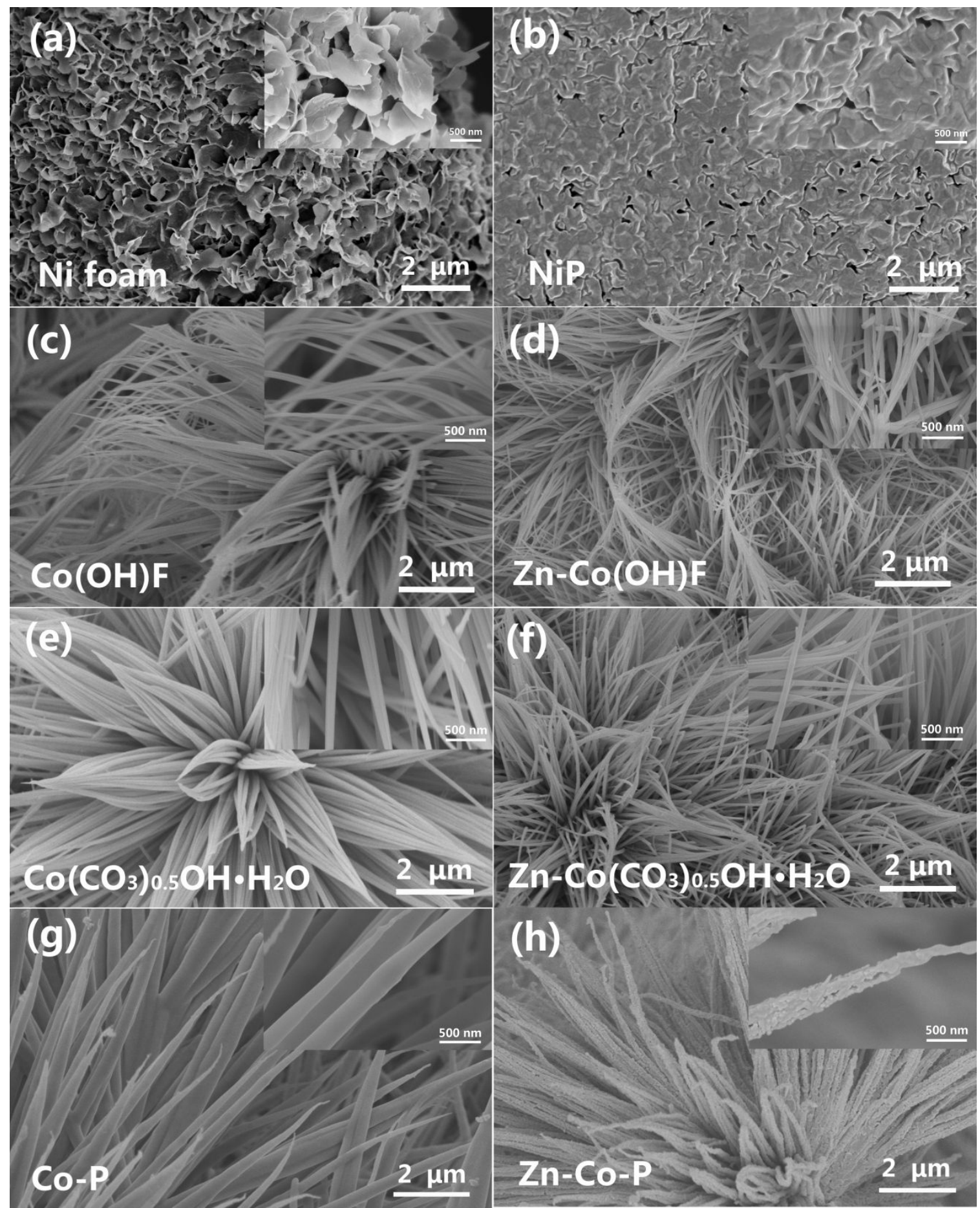

Figure S3. Surface morphology of the precursors in different stages of synthesis. SEM images of

(a) bare Ni foam, (b) NiP (the Ni foam after phosphorization), (c) $\mathrm{Co}(\mathrm{OH}) \mathrm{F}$, (d) $\mathrm{Zn}-\mathrm{Co}(\mathrm{OH}) \mathrm{F}$,

(e) $\mathrm{Co}\left(\mathrm{CO}_{3}\right)_{0.5} \mathrm{OH} \bullet 0.11 \mathrm{H}_{2} \mathrm{O}$, (f) $\mathrm{Zn}-\mathrm{Co}\left(\mathrm{CO}_{3}\right)_{0.5} \mathrm{OH} \bullet 0.11 \mathrm{H}_{2} \mathrm{O}$, (g) Co-P and (h) Zn-Co-P. 
Figure S3a-h demonstrates the typical SEM images of all the precursors in different stages during synthesis. Obviously, the pretreated bare Ni foam has a rough surface with large amounts of pits, and after phosphorization the pits shrink together to give a relatively flat surface (Figure S3a and b). The SEM images of $\mathrm{Co}(\mathrm{OH}) \mathrm{F}$ and $\mathrm{Zn}-\mathrm{Co}(\mathrm{OH}) \mathrm{F}$ are displayed in Figure S3c and d, demonstrating that the surface of $\mathrm{Ni}$ foam is uniformly coated with cluster-like nanowires with a diameter of $\sim 100 \mathrm{~nm}$ after the hydrothermal process. After thermal treatment, the appearance of these nanowires does not change obviously except the aggregation of the tips of the nanowires (Figure S3e and f). However, after the phosphorizaiton process, the nanowires become thick remarkably with a diameter of $\sim 200 \mathrm{~nm}$ (Figure S3g and h), which may be caused by the deposition of phosphorus. 


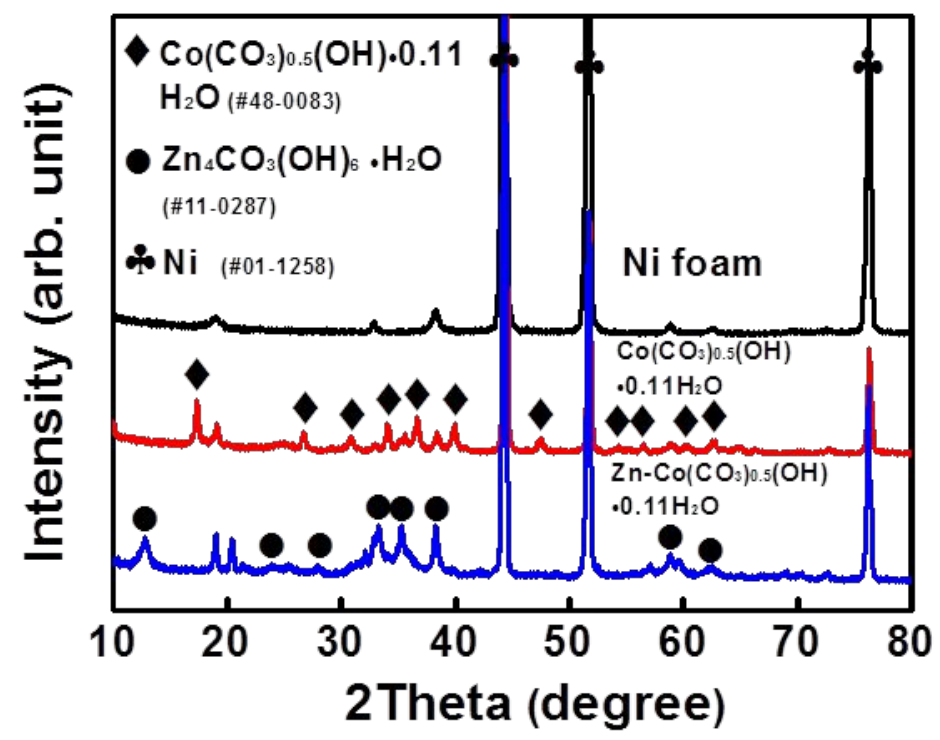

Figure S4. XRD patterns of bare $\mathrm{Ni}$ foam and the precursors of $\mathrm{Co}\left(\mathrm{CO}_{3}\right)_{0.5} \mathrm{OH} \cdot 0.11 \mathrm{H}_{2} \mathrm{O}$ and $\mathrm{Zn}$ $\mathrm{Co}\left(\mathrm{CO}_{3}\right)_{0.5} \mathrm{OH} \cdot 0.11 \mathrm{H}_{2} \mathrm{O}$.

In order to further investigate the structure of the precursors before phosphorization, XRD characterization was carried out on the thermal treated precursors. As depicted in Figure S4, the peaks of the precursor without $\mathrm{Zn}$ incorporation are well matched with the standard diffraction patterns of $\mathrm{Co}\left(\mathrm{CO}_{3}\right)_{0.5} \mathrm{OH} \cdot 0.11 \mathrm{H}_{2} \mathrm{O}$ (JCPDS no. 48-0083), meaning that the $\mathrm{Co}(\mathrm{OH}) \mathrm{F}$ precursor has transformed into $\mathrm{Co}\left(\mathrm{CO}_{3}\right)_{0.5} \mathrm{OH} \cdot 0.11 \mathrm{H}_{2} \mathrm{O}$ after thermal treatment. Meanwhile, there are only diffraction peaks indexed to $\mathrm{Zn}\left(\mathrm{CO}_{3}\right)_{0.5} \mathrm{OH} \cdot \mathrm{H}_{2} \mathrm{O}$ (JCPDS no. 11-0287) phase in the $\mathrm{Zn}$ incorporated precursor, demonstrating that most of the surface may be covered by the $\mathrm{Zn}\left(\mathrm{CO}_{3}\right)_{0.5} \mathrm{OH} \cdot \mathrm{H}_{2} \mathrm{O}$ component. 

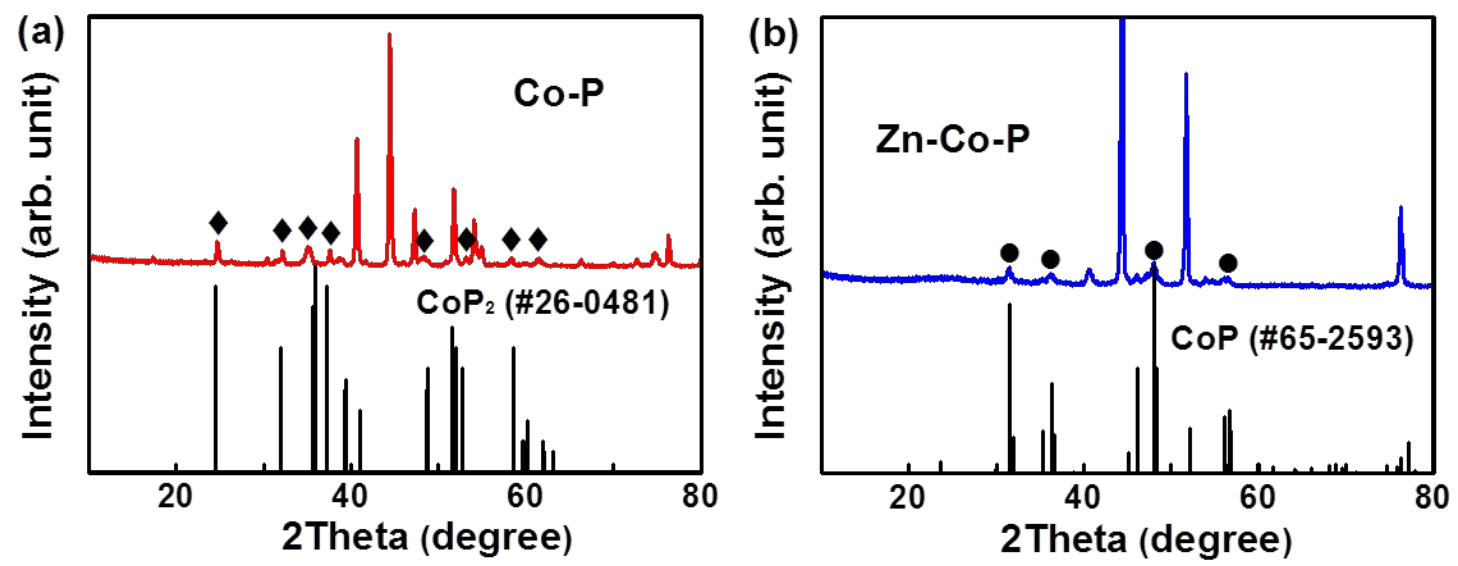

Figure S5. XRD patterns of (a) Co-P and (b) Zn-Co-P and the contrast with the corresponding PDF cards of $\mathrm{CoP}_{2}(\# 26-0481)$ and $\mathrm{CoP}(\# 65-2593)$ phases. 

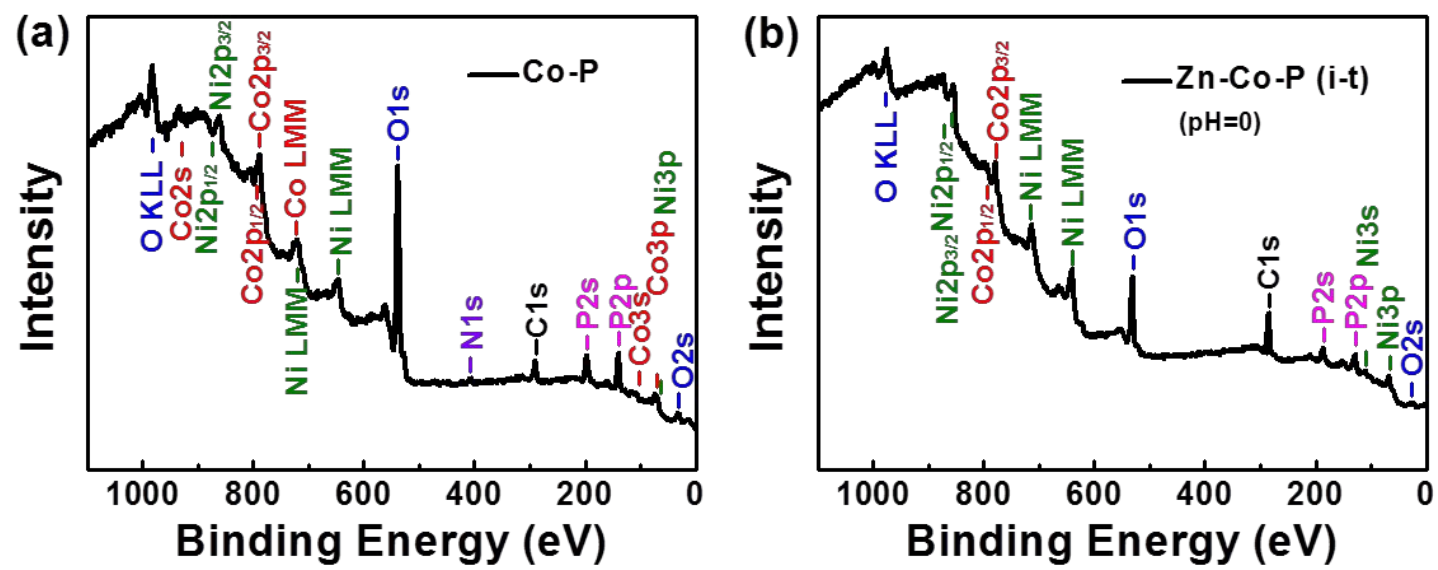

Figure S6. XPS survey spectra of (a) Co-P and (b) Zn-Co-P after the i-t test at the static overpotential of $170 \mathrm{mV}$ for $15 \mathrm{~h}$ in $0.5 \mathrm{M} \mathrm{H}_{2} \mathrm{SO}_{4}$. 

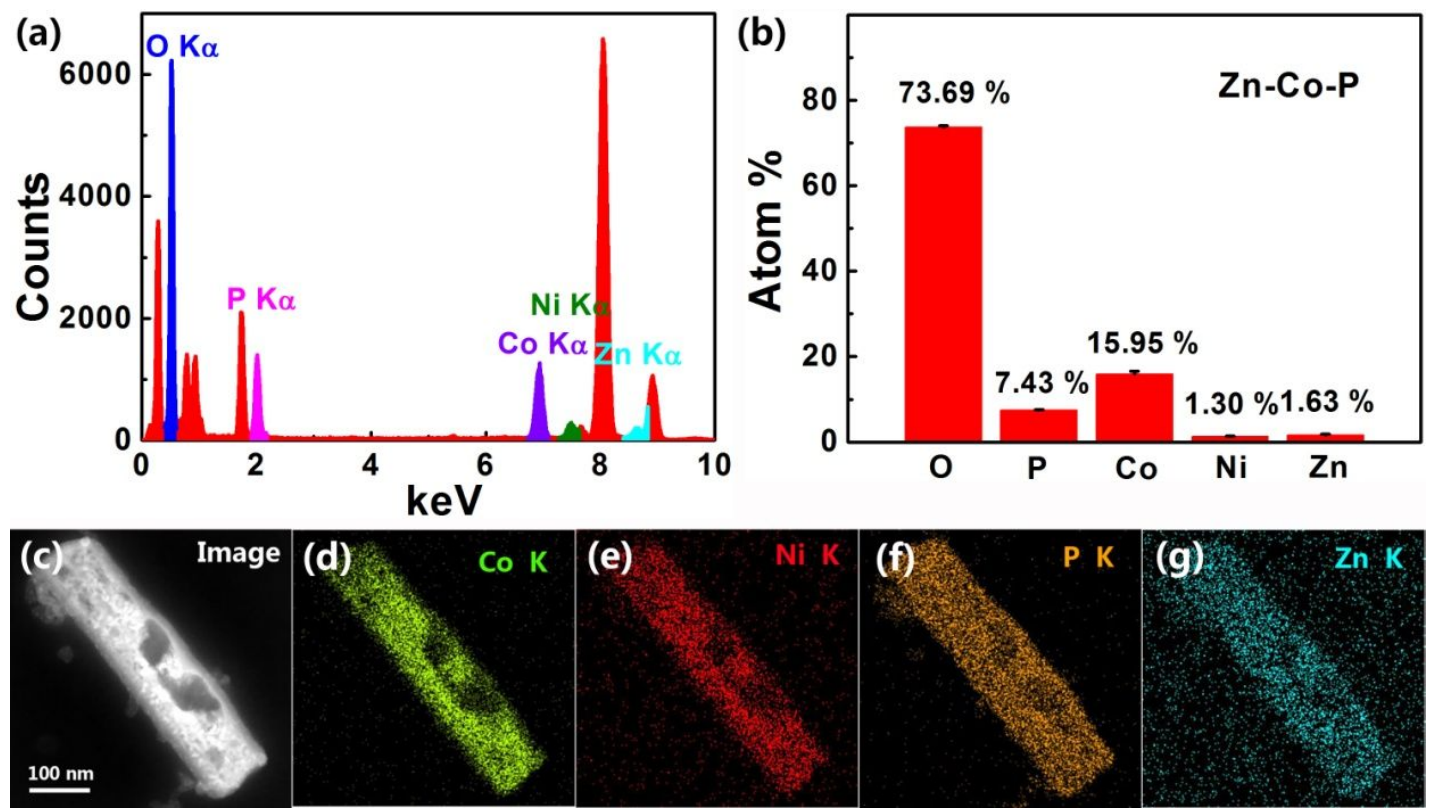

Figure S7. (a) Energy-dispersive X-ray spectrum (EDX) of a single Zn-Co-P nanowire and (b) the corresponding elemental atomic ratios of $\mathrm{Co}, \mathrm{Ni}, \mathrm{P}, \mathrm{O}$ and $\mathrm{Zn}$. (c-g) EDX elemental mapping images of $\mathrm{Co}, \mathrm{Ni}, \mathrm{P}$ and $\mathrm{Zn}$ in $\mathrm{Zn}-\mathrm{Co}-\mathrm{P}$.

In the EDX elemental mapping images (Figure S7d-g), Ni signals can also be detected in the $\mathrm{Zn}-\mathrm{Co}-\mathrm{P}$ nanowire demonstrating that $\mathrm{Ni}$ doping may take place in the synthesis process. During the hydrothermal step, the dipped Ni foam may dissolve slightly and enter into the growing nanowires. Though $\mathrm{Ni}$ doping has a positive effect on cobalt phosphides, this phenomenon exists in all the samples. Hence, it is still meaningful to compare the catalytic activities of Co-P and Zn-Co-P. 

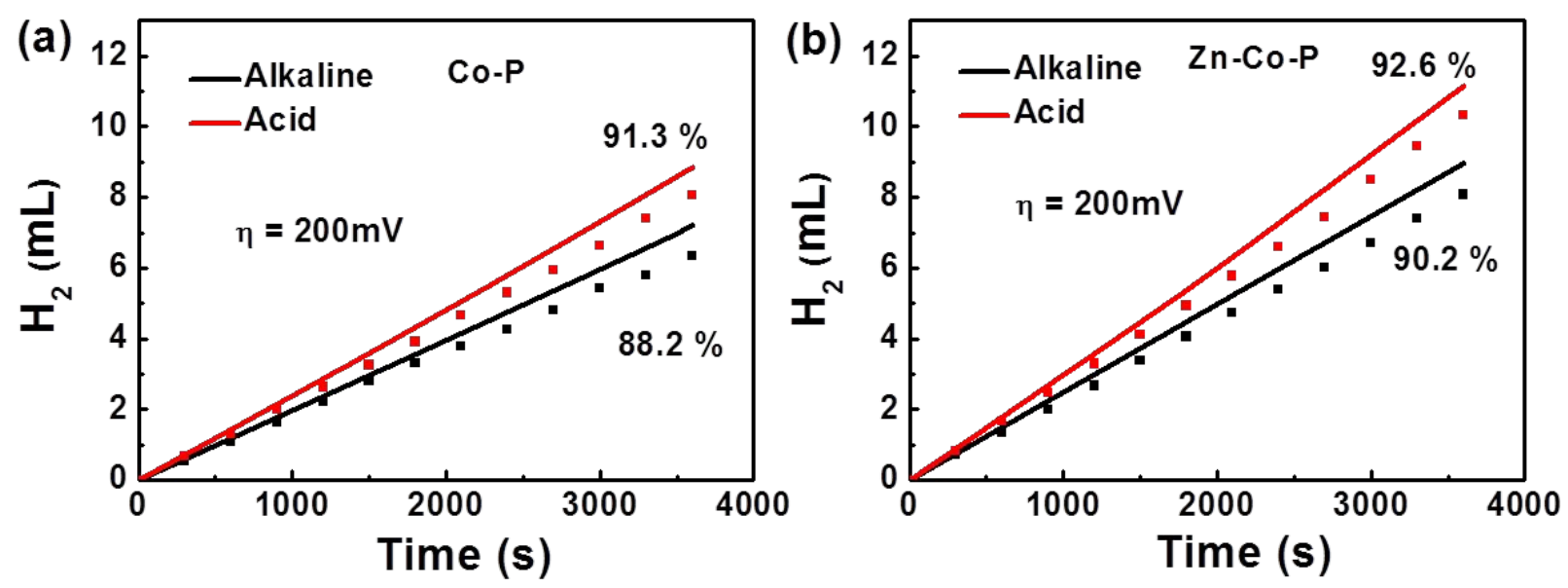

Figure S8. The volumes of calculated (line) and measured (scattered dots) hydrogen under the standard conditions vs. time for (a) Co-P and (b) Zn-Co-P at a constant overpotential of $200 \mathrm{mV}$ for $1 \mathrm{~h}$ in acidic and alkaline electrolytes, respectively. 

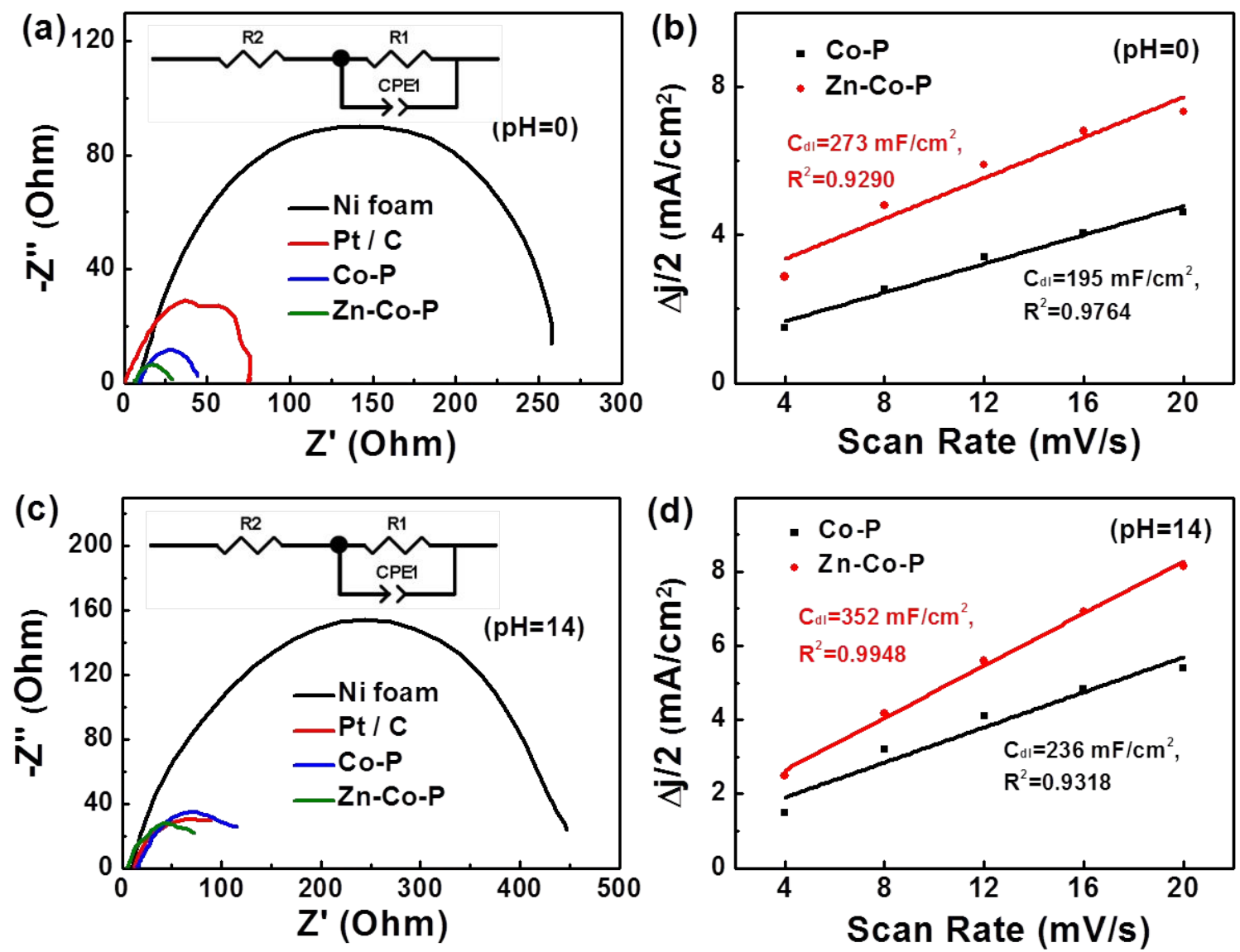

Figure S9. EIS and $\mathrm{C}_{\mathrm{dl}}$ measurements. Nyquist plots of bare Ni foam, Pt/C, Co-P and Zn-Co-P in (a) $0.5 \mathrm{M} \mathrm{H}_{2} \mathrm{SO}_{4}$ and (c) $1.0 \mathrm{M} \mathrm{KOH}$, respectively. The capacitive current density as a function of scanning rate for Co-P and $\mathrm{Zn}-\mathrm{Co}-\mathrm{P}$ in (b) $0.5 \mathrm{M} \mathrm{H}_{2} \mathrm{SO}_{4}$ and (d) $1.0 \mathrm{M} \mathrm{KOH}$. 

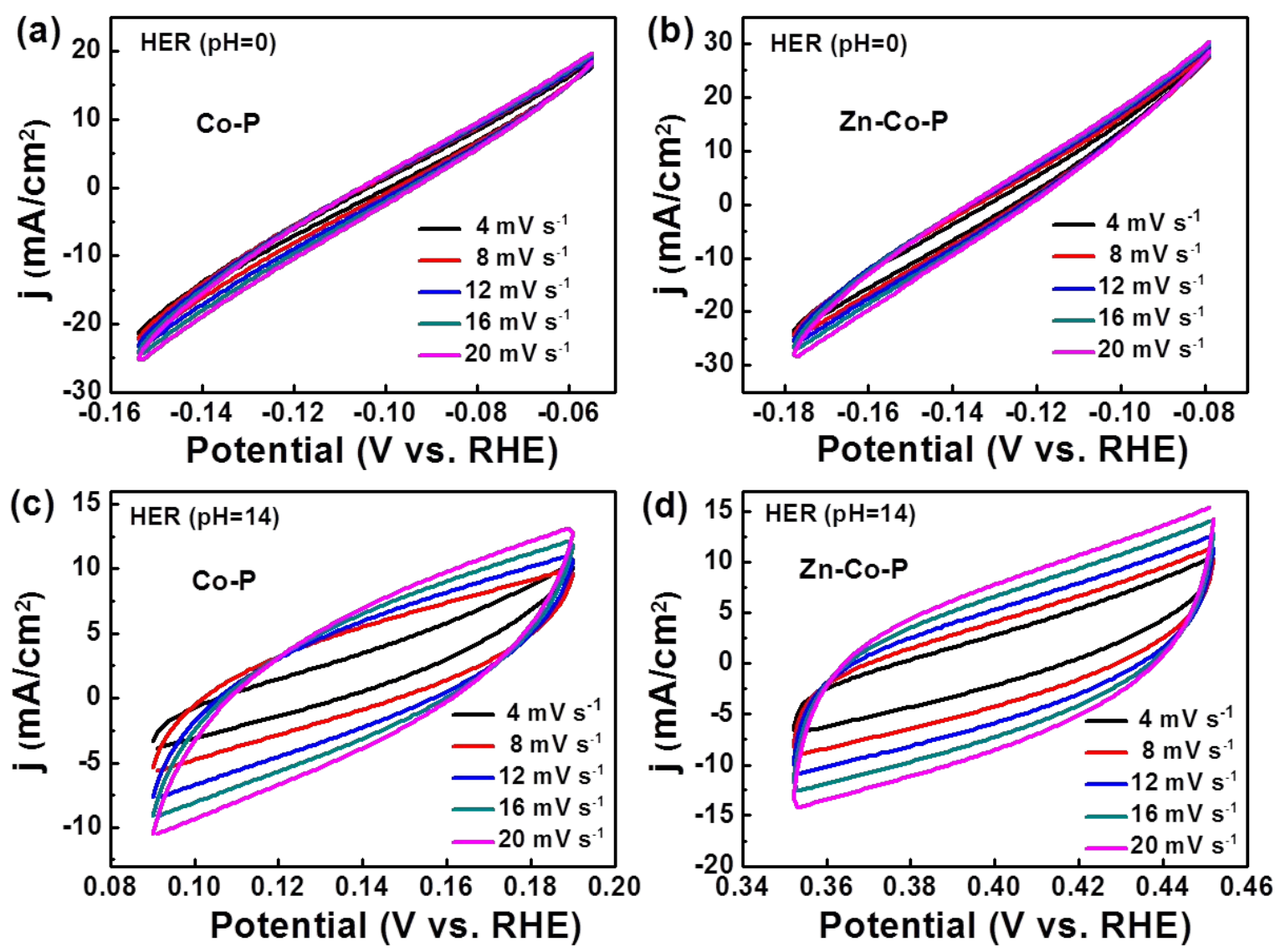

Figure S10. The CVs of Co-P and Zn-Co-P in (a, b) $0.5 \mathrm{M} \mathrm{H}_{2} \mathrm{SO}_{4}$ and (c, d) $1.0 \mathrm{M} \mathrm{KOH}$. 


\section{Electrochemical active surface area}

The electrochemical active surface area (ECSA) was determined by the electrochemical capacitance measurements. Cyclic voltammetry (CV) was used to investigate the double layer capacitance $\left(\mathrm{C}_{\mathrm{dl}}\right)$ and the sweeping range was determined by the open circuit potential. The potential was swept in the specified range six times at five different scan rates $(4,8,12,16,20$ $\mathrm{mV} / \mathrm{s}$ ). The measured cyclic voltammograms are shown in Figure $\mathbf{S 1 0}$ for Co-P and Zn-Co-P in two electrolytes $(\mathrm{pH}=0,14)$. The capacitive currents were measured in a potential where no faradic processes occur. The half value of the difference between the high and low currents was plotted as a function of scanning rate (Figure S9b and S9d) and a linear fit was carried out to determine the specific capacitance. Meanwhile, the obtained specific capacitance can be converted into the ECSA according to the specific capacitance value of a flat standard material with a real surface area of $1 \mathrm{~cm}^{2}$. The specific capacitance of a flat surface is generally in the range of $20 \sim 60 \mu \mathrm{F} \bullet \mathrm{cm}^{-2}{ }^{1-2}$ In this work, we assume $40 \mu \mathrm{F} \bullet \mathrm{cm}^{-2}$ as the standard capacitance value of the flat surface for the following calculations of ECSA and TOF.

Calculation of the ECSA

$$
\begin{aligned}
& \mathrm{pH}=0: \quad \mathrm{A}_{\mathrm{ECSA}}^{\mathrm{Co}}-\mathrm{P}=\frac{195000 \mu \mathrm{F} \bullet \mathrm{cm}^{-2}}{40 \mu \mathrm{F} \cdot \mathrm{cm}^{-2} \text { per } \mathrm{cm}_{\mathrm{ECSA}}^{2}}=4875 \mathrm{~cm}_{\mathrm{ECSA}}^{2} \\
& \mathrm{pH}=0: \quad \mathrm{A}_{\mathrm{ECSA}}^{\mathrm{Zn}-\mathrm{Co}-\mathrm{P}}=\frac{273000 \mu \mathrm{F} \cdot \mathrm{cm}^{-2}}{40 \mu \mathrm{F} \cdot \mathrm{cm}^{-2} \text { per } \mathrm{cm}_{\mathrm{ECSA}}^{2}}=6825 \mathrm{~cm}_{\mathrm{ECSA}}^{2} \\
& \mathrm{pH}=14: \quad \mathrm{A}_{\mathrm{ECSA}}^{\mathrm{Co}}-\mathrm{P}=\frac{236000 \mu \mathrm{F} \bullet \mathrm{cm}^{-2}}{40 \mu \mathrm{F} \cdot \mathrm{cm}^{-2} \text { per } \mathrm{cm}_{\mathrm{ECSA}}^{2}}=5900 \mathrm{~cm}_{\mathrm{ECSA}}^{2} \\
& \mathrm{pH}=14: \quad \mathrm{A}_{\mathrm{ECSA}}^{\mathrm{Zn}-\mathrm{Co}-\mathrm{P}}=\frac{352000 \mu \mathrm{F} \cdot \mathrm{cm}^{-2}}{40 \mu \mathrm{F} \cdot \mathrm{cm}^{-2} \text { per } \mathrm{cm}_{\mathrm{ECSA}}^{2}}=8800 \mathrm{~cm}_{\mathrm{ECSA}}^{2}
\end{aligned}
$$


Calculation of the TOF

The per-site TOF was calculated using the following formula:

$$
\mathrm{TOF}=\frac{\# \text { total hydrogen turn overs } / \mathrm{cm}^{2} \text { geometric area }}{\# \text { surface sites } / \mathrm{cm}^{2} \text { geometric area }}
$$

The total number of hydrogen turnovers was obtained from the geometric current density based on the following formula: ${ }^{3-4}$

$$
\begin{aligned}
& \# \mathrm{H}_{2}=\left(\mathrm{j} \frac{\mathrm{mA}}{\mathrm{cm}^{2}}\right) \cdot\left(\frac{1 \mathrm{C} \cdot \mathrm{s}^{-1}}{1000 \mathrm{~mA}}\right) \cdot\left(\frac{1 \mathrm{~mol} \mathrm{e}^{-}}{96485.3 \mathrm{C}}\right) \cdot\left(\frac{1 \mathrm{~mol} \mathrm{H}_{2}}{2 \mathrm{~mol} \mathrm{e}^{-}}\right) \cdot\left(\frac{6.022 \times 10^{23} \mathrm{H}_{2} \mathrm{molecules}}{1 \mathrm{~mol} \mathrm{H}_{2}}\right)=3.12 \\
& \times 10^{15} \frac{\mathrm{H}_{2} / \mathrm{s}}{\mathrm{cm}^{2}} \operatorname{per} \frac{\mathrm{mA}}{\mathrm{cm}^{2}}
\end{aligned}
$$
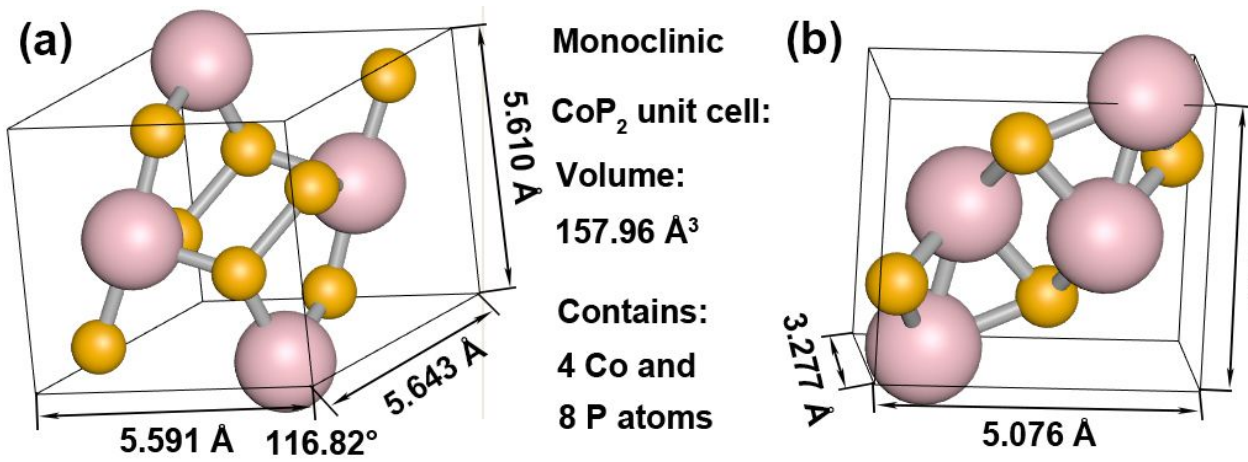

Orthorhombic

CoP unit cell:

Volume:

or $93.13 \AA^{3}$

کुํำ

Contains:

4 Co and

$4 \mathrm{P}$ atoms

Figure S11. (a) $\mathrm{CoP}_{2}$ and (b) $\mathrm{CoP}$ unit cell. Co atoms: pink and $\mathrm{P}$ atoms: yellow.

As the exact hydrogen bonding sites are not clear, we assume conservatively that all the exposed atoms including both Co and $\mathrm{P}$ atoms are the active sites. According to the phases of Co-P and $\mathrm{Zn}-\mathrm{Co}-\mathrm{P}$, the total number of surface sites were estimated from the unit cells of $\mathrm{CoP}_{2}$ and $\mathrm{CoP}$ in Figure S11, respectively. ${ }^{5-6}$

\# surface sites per real surface area (for $\left.\mathrm{CoP}_{2}\right)$ : 


$$
\text { \#surface sites }=\left(\frac{12 \text { atoms/unit cell }}{157.96 \AA^{3} / \text { unit cell }^{2}}\right)^{\frac{2}{3}}=1.794 \times 10^{15} \text { atoms } \bullet \mathrm{cm}_{\text {real }}^{-2}
$$

\# surface sites per real surface area (for $\mathrm{CoP})$ :

$$
\text { \#surface sites }=\left(\frac{8 \text { atoms } / \text { unit cell }}{93.13 \AA^{3} / \text { unit cell }^{\frac{2}{3}}}\right)^{\frac{1}{3}}=1.947 \times 10^{15} \text { atoms } \bullet \mathrm{cm}_{\text {real }}^{-2}
$$

Finally, the TOF plot can be obtained from the plot of current density according to the following formula:

$$
\mathrm{TOF}=\frac{\left(3.12 \times 10^{15} \frac{\mathrm{H}_{2} / \mathrm{s}}{\mathrm{cm}^{2}} \text { per } \frac{\mathrm{mA}}{\mathrm{cm}^{2}}\right) \times|\mathrm{j}|}{\# \text { surface sites } \times \mathrm{A}_{\mathrm{ECSA}}}
$$

The polarization curves and TOF plots of Co-P and $\mathrm{Zn}-\mathrm{Co}-\mathrm{P}$ in three electrolytes are shown in Figure S12. 

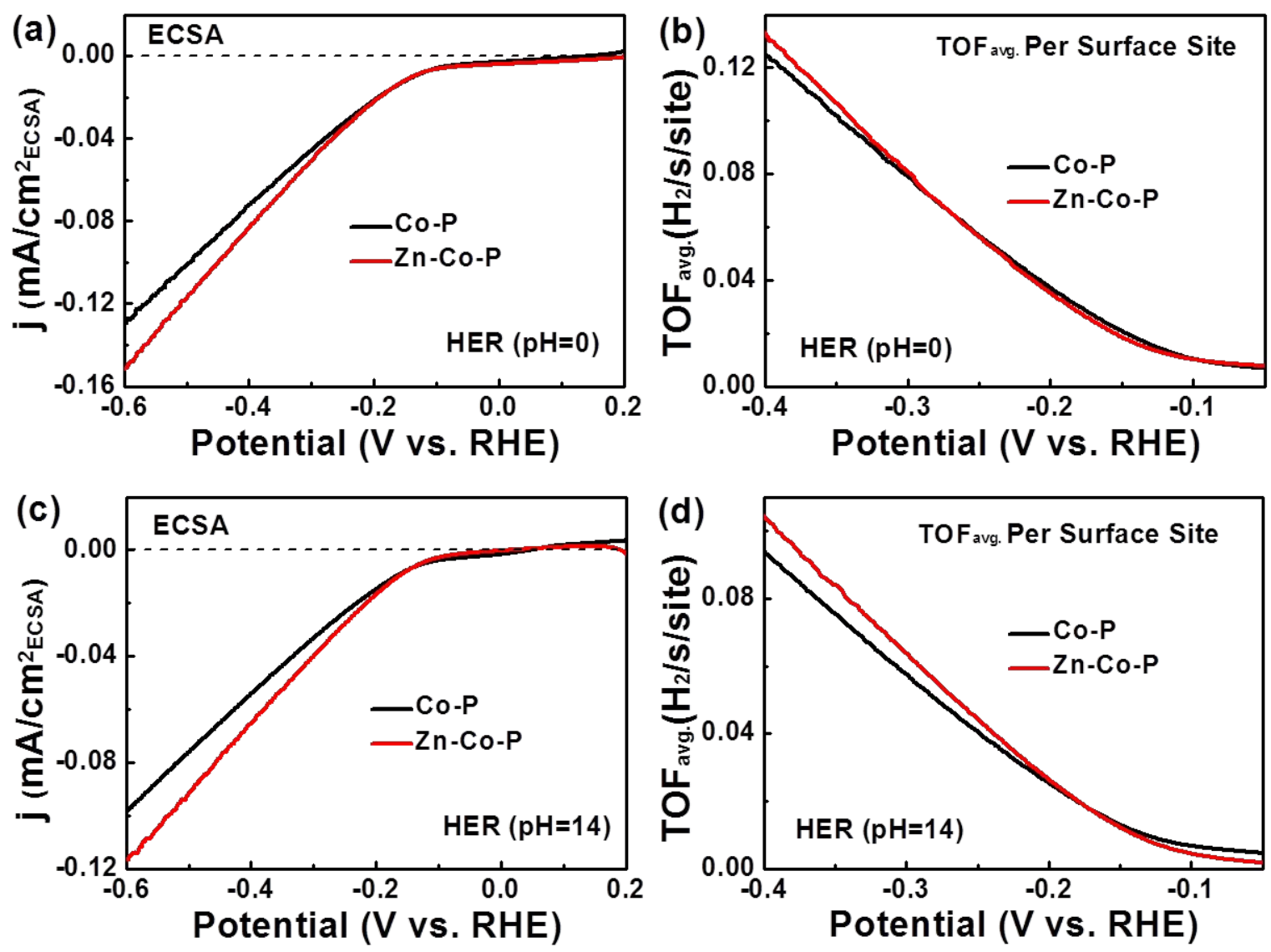

Figure S12. The polarization curves normalized to the ECSA and TOF versus overpotential plots using $40 \mu \mathrm{F} \cdot \mathrm{cm}^{-2}$ as the standard specific capacitance value of Co-P and Zn-Co-P in (a, b) $0.5 \mathrm{M}$ $\mathrm{H}_{2} \mathrm{SO}_{4}$ and (c, d) $1.0 \mathrm{M} \mathrm{KOH}$. 

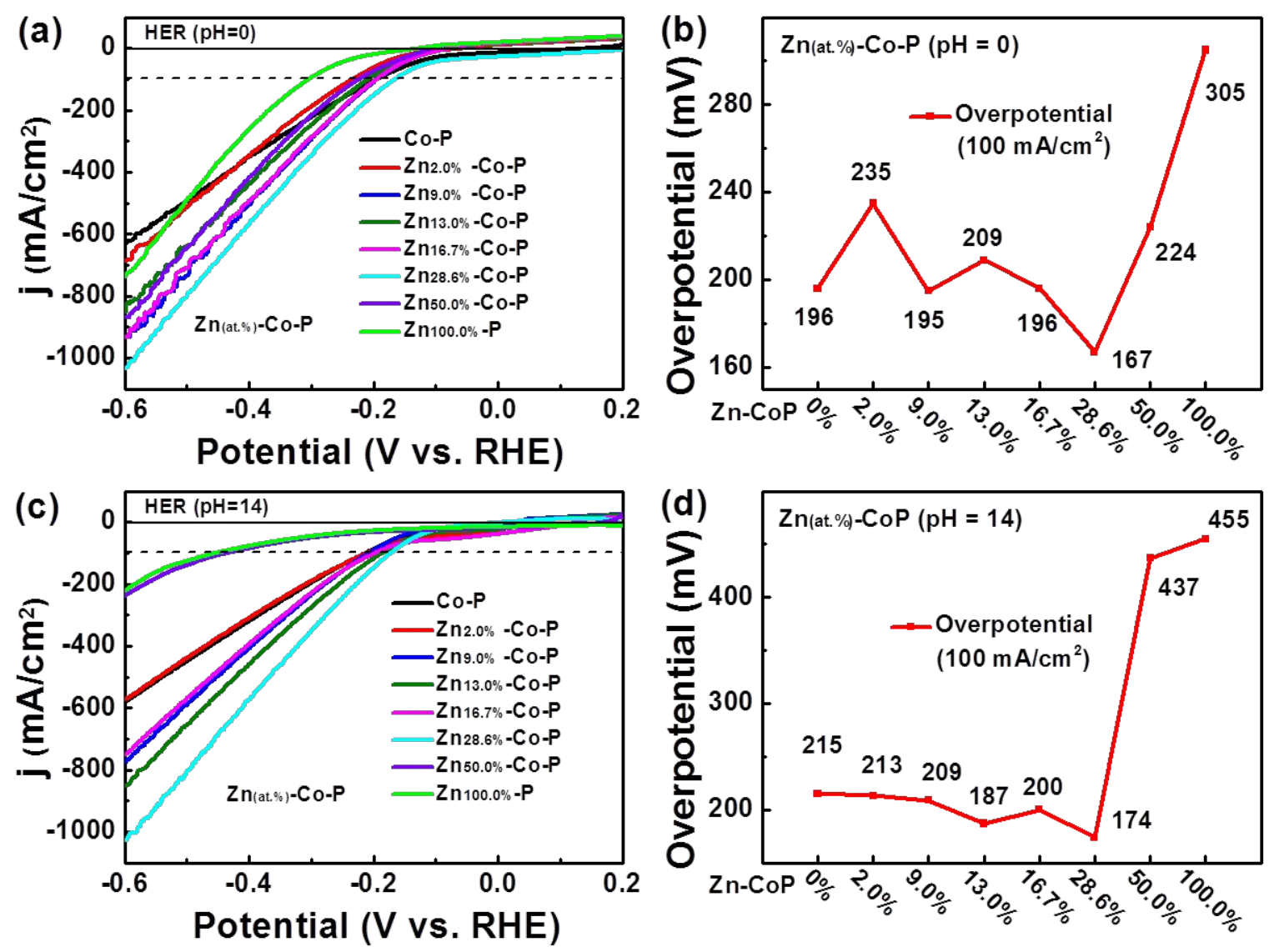

Figure S13. HER performance of $\mathrm{Zn}_{(\mathrm{at.} \%)}$-Co-P catalysts in acidic and alkaline conditions. (a, c) Polarization curves and (b, d) the $\mathrm{Zn}$ dopant concentration dependent HER activities of $\mathrm{Zn}_{(\text {at. \%)- }}$ Co-P catalysts. 


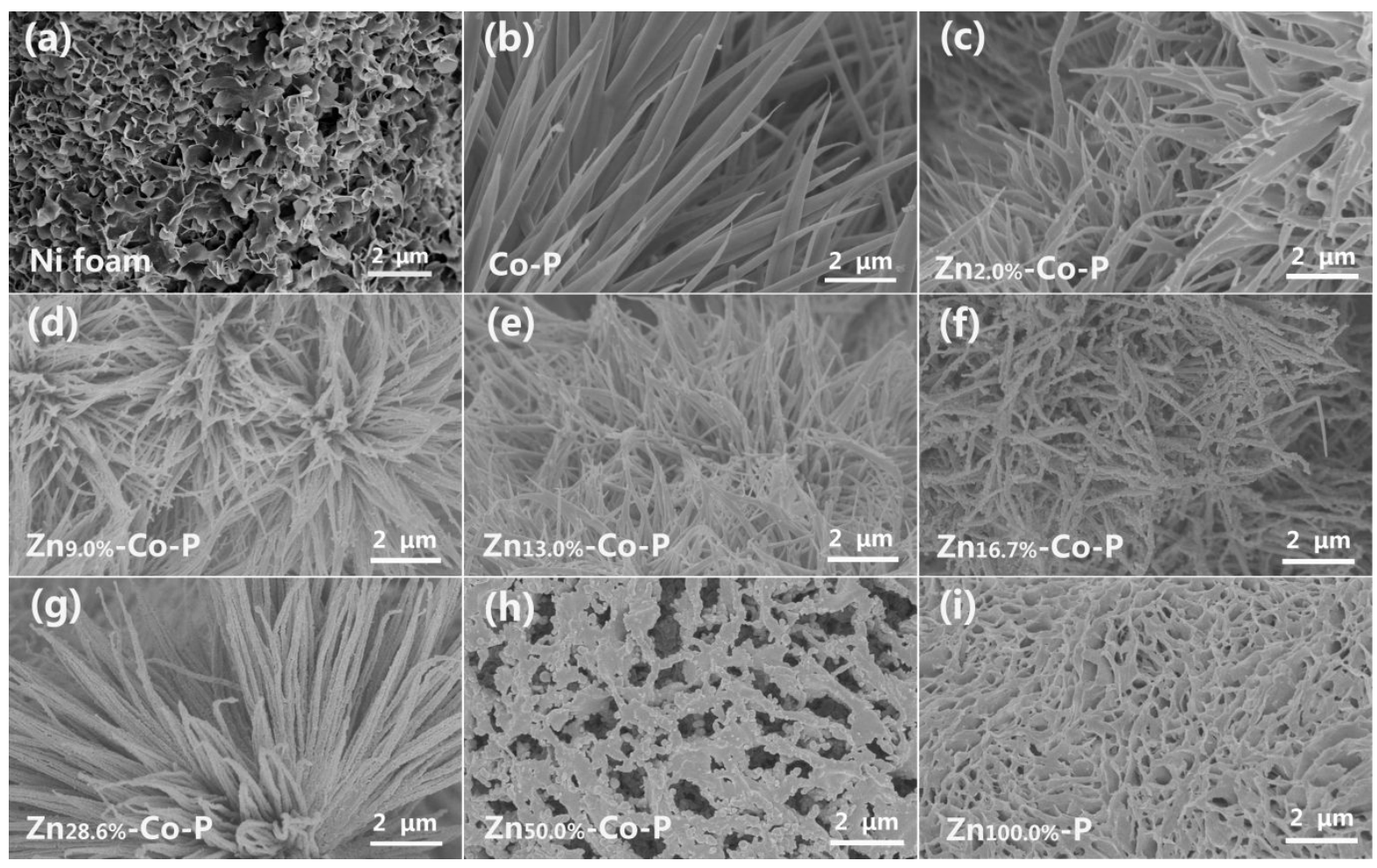

Figure S14. The Zn dopant concentration dependent surface morphology. (a-i) SEM images of bare Ni foam, Co-P, $\mathrm{Zn}_{(\text {at. \%) }}-\mathrm{Co}-\mathrm{P}$ and $\mathrm{Zn}-\mathrm{P}$. 


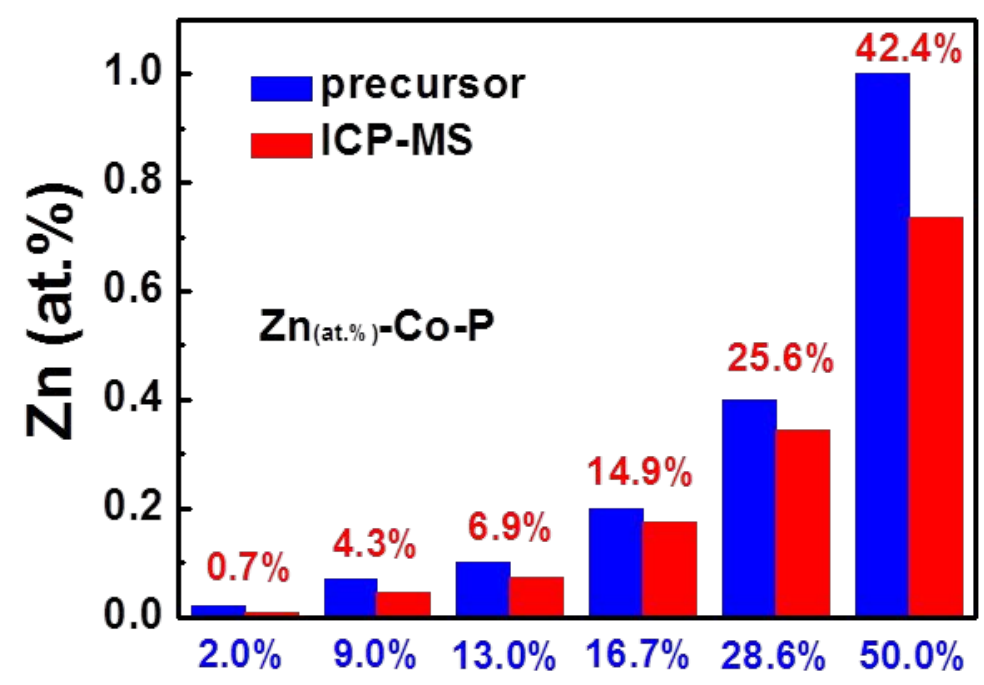

Figure S15. The atomic ratios of $\mathrm{Zn}$ element in the $\mathrm{Zn}_{(\mathrm{at} .}$ \%)- $\mathrm{Co}(\mathrm{OH}) \mathrm{F}$ precursors determined by ICP-MS (blue column: the ratio of the added $\mathrm{Zn}$ element during the synthesis, red column: the ratio measured by ICP-MS). 

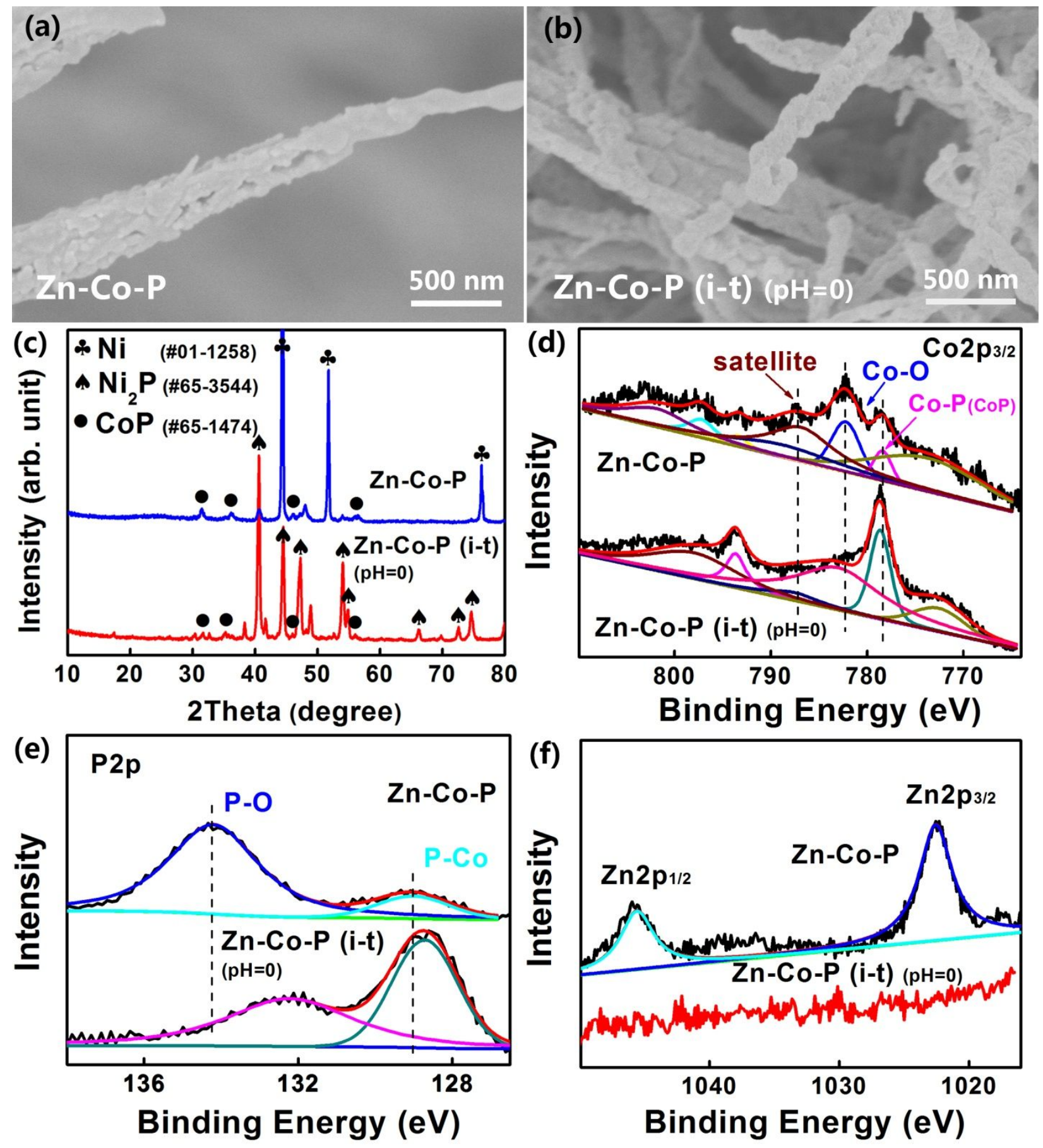

Figure S16. The morphological and structural characterizations of $\mathrm{Zn}-\mathrm{Co}-\mathrm{P}$ before and after the i-t test in acidic condition. (a-b) SEM images, (c) XRD patterns, and (d-f) the high-resolution XPS spectra of (d) Co 2p, (e) P 2p, and (f) Zn 2p of Zn-Co-P before and after the i-t test at the static overpotential of $170 \mathrm{mV}$ for stability test in $0.5 \mathrm{M} \mathrm{H}_{2} \mathrm{SO}_{4}$ for $15 \mathrm{~h}$. 

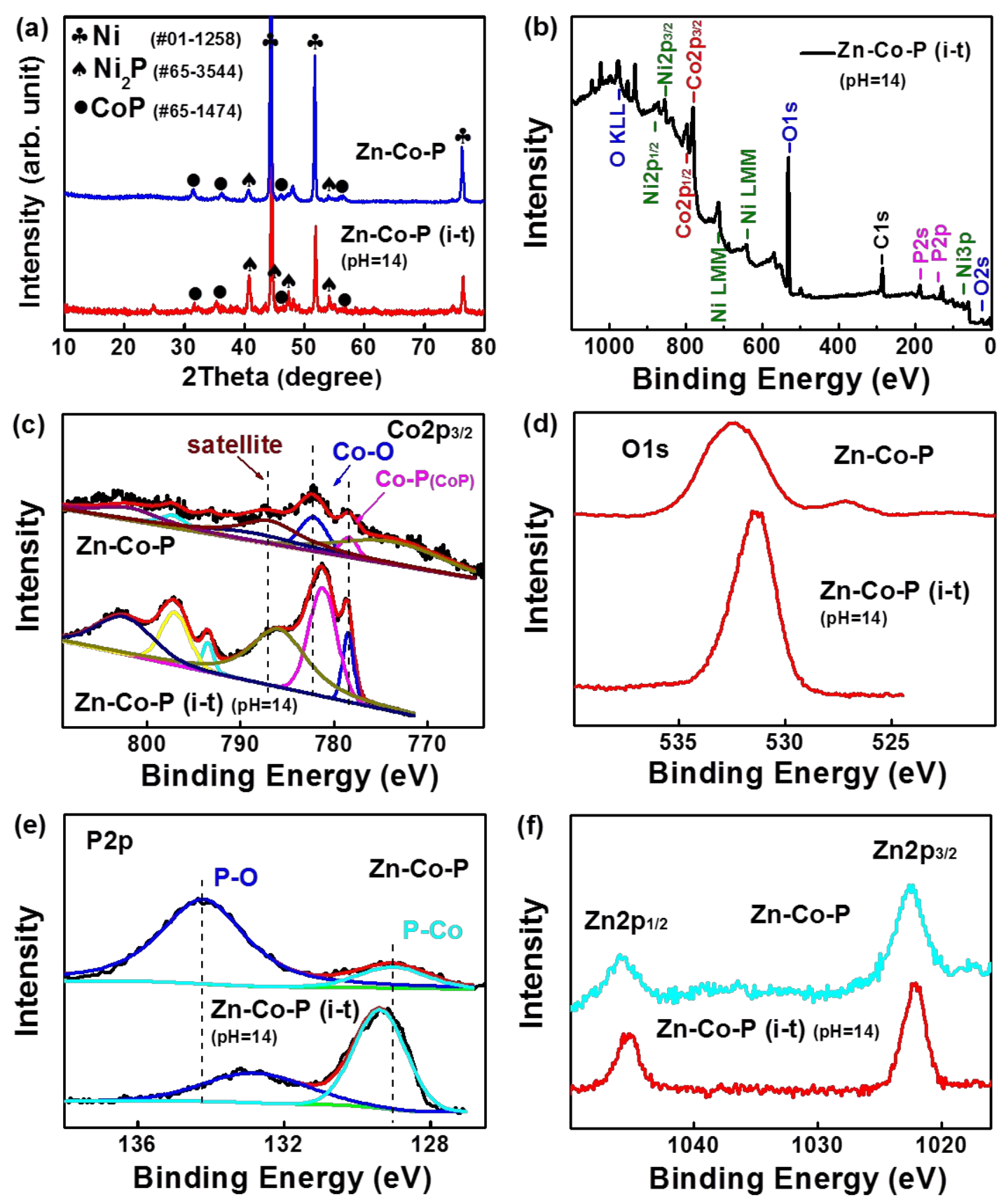

Figure S17. Structural characterizations of $\mathrm{Zn}-\mathrm{Co}-\mathrm{P}$ before and after the i-t test in alkaline condition. (a) XRD patterns, (b) survey spectrum and (c-f) high-resolution XPS spectra of (c) Co 2p, (d) O 1s, (e) P 2p, and (f) Zn 2p of Zn-Co-P before and after the i-t test at the static overpotential of $180 \mathrm{mV}$ in $1.0 \mathrm{M} \mathrm{KOH}$ for $15 \mathrm{~h}$. 


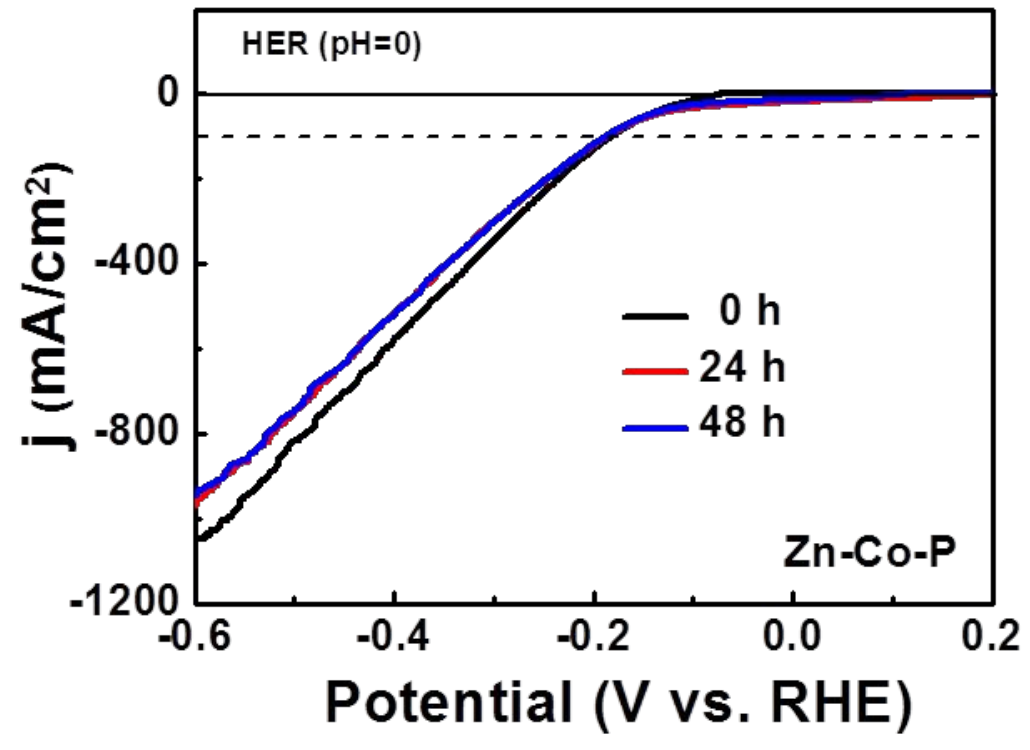

Figure S18. Polarization curves of Zn-Co-P in acidic media after acid dip for $24 \mathrm{~h}$ and $48 \mathrm{~h}$, respectively. 
Table S1. The quality values of all the samples at each step. The size of all the samples is $4 \mathrm{~cm}$ $\times 1 \mathrm{~cm}$, and the geometric area is $4 \mathrm{~cm}^{2}$.

\begin{tabular}{ccccc}
\hline Sample name & Ni foam & Zn-Co(OH)F & $\begin{array}{c}\mathrm{Zn}-\mathrm{Co}\left(\mathrm{CO}_{3}\right)_{0.5} \mathrm{OH} \bullet \\
0.11 \mathrm{H}_{2} \mathrm{O}\end{array}$ & Zn-Co-P \\
\hline Co-P & $140.8 \mathrm{mg}$ & $215.4 \mathrm{mg}$ & $209.8 \mathrm{mg}$ & $245.5 \mathrm{mg}$ \\
$\mathrm{Zn}_{2.0 \%}$-Co-P & $140.1 \mathrm{mg}$ & $212.8 \mathrm{mg}$ & $208.8 \mathrm{mg}$ & $233.6 \mathrm{mg}$ \\
$\mathrm{Zn}_{9.0 \%}$-Co-P & $140.2 \mathrm{mg}$ & $231.3 \mathrm{mg}$ & $224.4 \mathrm{mg}$ & $250.9 \mathrm{mg}$ \\
$\mathrm{Zn}_{13.0 \%}-\mathrm{Co}-\mathrm{P}$ & $139.4 \mathrm{mg}$ & $223.1 \mathrm{mg}$ & $217.2 \mathrm{mg}$ & $249.6 \mathrm{mg}$ \\
$\mathrm{Zn}_{16.7 \%}$-Co-P & $139.9 \mathrm{mg}$ & $229.8 \mathrm{mg}$ & $225.6 \mathrm{mg}$ & $254.9 \mathrm{mg}$ \\
$\mathrm{Zn}_{28.6 \%}-\mathrm{Co}-\mathrm{P}$ & $141.2 \mathrm{mg}$ & $221.8 \mathrm{mg}$ & $216.1 \mathrm{mg}$ & $252.6 \mathrm{mg}$ \\
$\mathrm{Zn}_{50.0 \%}$-Co-P & $141.8 \mathrm{mg}$ & $247.0 \mathrm{mg}$ & $255.8 \mathrm{mg}$ & $281.6 \mathrm{mg}$ \\
$\mathrm{Zn}_{100 . \%}-\mathrm{P}$ & $140.7 \mathrm{mg}$ & $232.7 \mathrm{mg}$ & $242.8 \mathrm{mg}$ & $246.2 \mathrm{mg}$ \\
\hline
\end{tabular}

\footnotetext{
* The value is obtained by the weight difference method.
} 
Table S2 Comparison of the HER electrochemical activity parameters between different catalysts reported recently. Some of the information were not specified and were estimated by the given graphs. The electrolyte is $0.5 \mathrm{M} \mathrm{H}_{2} \mathrm{SO}_{4}$.

\begin{tabular}{|c|c|c|c|c|c|}
\hline Catalysts & $\begin{array}{l}\eta_{10} / \\
\mathrm{mV}\end{array}$ & $\begin{array}{l}\eta_{100} / \\
\mathrm{mV}\end{array}$ & $\begin{array}{l}\text { Tafel } \\
\text { slope }\end{array}$ & Notes & Reference \\
\hline $\begin{array}{l}\mathrm{Zn-Co-P@Ni} \\
\text { foam }\end{array}$ & & 167 & 170 & $\begin{array}{l}\text { Zn-incorporated microporous CoP } \\
\text { nanowires array on nickel foam }\end{array}$ & This work \\
\hline $\mathrm{Zn}_{0.08} \mathrm{Co}_{0.92} \mathrm{P} / \mathrm{TM}$ & 39 & 83 & 39 & $\begin{array}{l}\text { Zn-doped CoP nanowall arrays on } \\
\text { titanium mesh }\end{array}$ & $\begin{array}{c}A d v . \text { Energy Mater. } \\
\mathbf{2 0 1 7}, 7(15) \\
1700020\end{array}$ \\
\hline CC@N-CoP & 42 & 78 & 41.2 & $\begin{array}{l}\text { N-doped CoP nanowires grown on } \\
\text { carbon cloth }\end{array}$ & $\begin{array}{c}\text { Adv. Mater. 2018, } \\
\text { 30, } 1800140\end{array}$ \\
\hline $\mathrm{CoP} / \mathrm{CC}$ & 49 & 94 & 30.1 & $\begin{array}{l}\text { Rugae-like structure } \\
\text { on carbon cloth }\end{array}$ & $\begin{array}{c}\text { Nano Energy 2015, } \\
\text { 15, 634-641 }\end{array}$ \\
\hline $\mathrm{CoP}_{2} / \mathrm{CC}-4$ & & 245 & 67 & $\begin{array}{l}\text { Mesoporous } \mathrm{CoP}_{2} \text { nanowires on } \\
\text { carbon cloth by alkaline etching }\end{array}$ & $\begin{array}{l}\text { J. Mater. Chem. A } \\
\text { 2018, } 6(39), \\
\text { 19038-19046 }\end{array}$ \\
\hline $\mathrm{CoP} / \mathrm{Ni}_{5} \mathrm{P}_{4} / \mathrm{CoP}$ & & 84 & 55 & $\begin{array}{l}\text { Hierarchical } \mathrm{CoP} / \mathrm{Ni}_{5} \mathrm{P}_{4} / \mathrm{CoP} \\
\text { microsheet arrays on } \mathrm{Ni} \text { foam }\end{array}$ & $\begin{array}{l}\text { Energ. Environ. } \\
\text { Sci. 2018, } 11(8), \\
\quad 2246-2252\end{array}$ \\
\hline $\begin{array}{c}\mathrm{Cu}-\mathrm{CoP} \\
\text { NRA/CC }\end{array}$ & 44 & & 86 & $\begin{array}{l}\mathrm{Cu} \text {-Doped CoP Nanorod Arrays } \\
\text { grown on carbon cloth }\end{array}$ & $\begin{array}{c}\text { ACS Appl. Energy } \\
\text { Mater. 2018, } 1 \text { (8), } \\
\text { 3835-3842 }\end{array}$ \\
\hline $\mathrm{Mn}-\mathrm{Co}-\mathrm{P} / \mathrm{Ti}$ & 49 & 105 & 55 & $\begin{array}{c}\text { Mn Doping of CoP Nanosheets Array } \\
\text { on Ti mesh }\end{array}$ & $\begin{array}{c}\text { ACS Catal. 2016, } \\
7(1), 98-102\end{array}$ \\
\hline $\mathrm{Fe}_{0.5} \mathrm{Co}_{0.5} \mathrm{P} / \mathrm{CC}$ & 37 & 98 & 30 & $\begin{array}{l}\text { Ternary } \mathrm{Fe}_{\mathrm{x}} \mathrm{Co}_{1-\mathrm{x}} \mathrm{P} \text { Nanowire Array on } \\
\text { carbon cloth }\end{array}$ & $\begin{array}{l}\text { Nano Lett. 2016, } \\
16(10), 6617-6621\end{array}$ \\
\hline \multirow{2}{*}{ CoP UPNSs } & & 130 & 44 & Sub-1.1 nm Ultrathin & Chem. Sci. 2017, \\
\hline & & & & Porous CoP Nanosheet & $8(4), 2769-2775$ \\
\hline $\mathrm{CoP} / \mathrm{CFP}-\mathrm{H}$ & $\begin{array}{c}128 . \\
1\end{array}$ & $\begin{array}{c}195 . \\
0\end{array}$ & 49.7 & $\begin{array}{l}\text { Nanostructuring Cobalt Phosphide } \\
\text { (CoP) Particles on Carbon Fiber Paper }\end{array}$ & $\begin{array}{c}\text { ACS Appl. Mater. } \\
\text { Inter. 2018, } 10(17), \\
14777-14785\end{array}$ \\
\hline
\end{tabular}




\begin{tabular}{|c|c|c|c|c|c|}
\hline $\mathrm{p}-\mathrm{CoP} / \mathrm{CP}$ & 38 & 136 & 52 & $\begin{array}{c}\text { Porous CoP nanosheets }(5 \sim 7 \mathrm{~nm}) \text { on } \\
\text { carbon paper }\end{array}$ & $\begin{array}{c}\text { Chem. Commun. } \\
\text { 2018, } 54 \text { (12), } \\
1465-1468\end{array}$ \\
\hline $\mathrm{V}-\mathrm{CoP} / \mathrm{CC}$ & & 120 & 54.9 & $\begin{array}{l}\text { V-CoP nanoneedle } \\
\text { arrays on } \mathrm{CC}\end{array}$ & $\begin{array}{l}\text { Chem. Sci. 2018, } \\
9(7), 1970-1975\end{array}$ \\
\hline $\begin{array}{l}\text { PANI/CoP } \\
\text { HNWs-CFs }\end{array}$ & 57 & 122 & 34.5 & $\begin{array}{l}\text { Polyaniline nanodots decorated CoP } \\
\text { hybrid nanowires on carbon fibers }\end{array}$ & $\begin{array}{l}\text { J. Am. Chem. Soc. } \\
\text { 2018, } 140(15) \text {, } \\
5118-5126\end{array}$ \\
\hline $\mathrm{CoPS} / \mathrm{CP}$ & 25.9 & 76.8 & 42.6 & $\begin{array}{c}\text { Cobalt phosphosulfide nanoneedles in } \\
\text { tetragonal phase supported on carbon } \\
\text { paper }\end{array}$ & 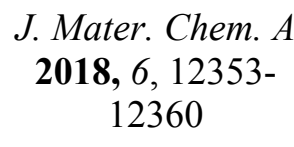 \\
\hline CoP@NPMG & 91 & & 58 & $\begin{array}{l}\text { CoP nanoparticles anchored on N,P- } \\
\text { dual-doped graphene-like carbon }\end{array}$ & $\begin{array}{l}\text { Nanoscale 2018, } \\
10(5), 2603-2612\end{array}$ \\
\hline $\mathrm{NiCo}_{2} \mathrm{P}_{\mathrm{x}} / \mathrm{CF}$ & 104 & & 59.6 & $\begin{array}{l}\text { Self-supported } \mathrm{NiCo}_{2} \mathrm{P}_{x} \text { nanowire } \\
\text { arrays on commercial carbon felt }\end{array}$ & $\begin{array}{l}\text { Adv. Mater. 2017, } \\
29(9), 1605502\end{array}$ \\
\hline
\end{tabular}

* The value is obtained from the polarization curves shown in the literatures. 
Table S3 Comparison of the HER electrochemical activity parameters between different catalysts reported recently. Some of the information were not specified and were estimated by the given graphs. The electrolyte is $1.0 \mathrm{M} \mathrm{KOH}$.

\begin{tabular}{|c|c|c|c|c|c|}
\hline Catalysts & $\begin{array}{l}\eta_{10} / \\
\mathrm{mV}\end{array}$ & $\begin{array}{l}\eta_{100} / \\
\mathrm{mV}\end{array}$ & $\begin{array}{l}\text { Tafel } \\
\text { slope }\end{array}$ & Notes & Reference \\
\hline $\begin{array}{l}\text { Zn-Co-P } \\
@ \text { Ni foam }\end{array}$ & & 172 & 116 & $\begin{array}{l}\mathrm{Zn} \text {-incorporated microporous CoP } \\
\text { nanowires array on nickel foam }\end{array}$ & This work \\
\hline CoP-A & & 245 & 76 & $\begin{array}{c}\text { hybrid } \mathrm{Co}(\mathrm{OH})_{\mathrm{x}} @ \mathrm{CoP} \text { nanosheets } \\
\text { by electrochemical activation }\end{array}$ & Chem. Sci. 2019 \\
\hline $\begin{array}{c}\mathrm{Fe}- \\
\mathrm{CoP} / \mathrm{NF}\end{array}$ & & 170 & 92 & $\begin{array}{l}\text { Fe doped CoP nanospheres } \\
\text { supported on Ni foam }\end{array}$ & $\begin{array}{l}A d v . S c i . \mathbf{2 0 1 8} \\
5(10), 1800949\end{array}$ \\
\hline $\begin{array}{l}\mathrm{Zn}_{0.08} \mathrm{Co}_{0.9} \\
{ }_{2} \mathrm{P} / \mathrm{TM}\end{array}$ & 67 & & 39 & $\begin{array}{l}\text { Zn-doped CoP nanowall array } \\
\text { supported on titanium mesh }\end{array}$ & $\begin{array}{l}\text { Adv. Energy Mater. } \\
\text { 2017, } 7 \text { (15), } 1700020\end{array}$ \\
\hline $\begin{array}{l}\text { CoP-400- } \\
\text { E15 film }\end{array}$ & 86 & 275 & 73 & $\begin{array}{l}\text { Micro-nanoporous } \mathrm{Co}_{2} \mathrm{P}-\mathrm{Co} \text { film } \\
\text { synthesized by } 400{ }^{\circ} \mathrm{C} \text { annealing } \\
\text { and } 15 \text { min } \mathrm{HCl} \text { etching from } \mathrm{CoP} \\
\text { amorphous film }\end{array}$ & $\begin{array}{l}\text { Adv. Energy Mater. } \\
\text { 2018, } 8(34), 1802445\end{array}$ \\
\hline $\begin{array}{l}\text { Co-B-P } \\
/ \mathrm{NF}\end{array}$ & 42 & & 42.1 & $\begin{array}{c}\text { Amorphous Co-B-P nanosheets } \\
\text { on Ni foam by electroless } \\
\text { deposition }\end{array}$ & $\begin{array}{c}\text { J. Mater. Chem. A 2018, } \\
\text { 6, 22062-22069 }\end{array}$ \\
\hline $\begin{array}{l}\mathrm{Cu}-\mathrm{CoP} \\
\mathrm{NRA} / \mathrm{CC}\end{array}$ & 81 & & 102 & $\begin{array}{l}\text { Cu-Doped CoP Nanorod Arrays } \\
\text { grown on carbon cloth }\end{array}$ & $\begin{array}{c}\text { ACS Appl. Energy } \\
\text { Mater. 2018, } 1 \text { (8), 3835- } \\
3842\end{array}$ \\
\hline $\begin{array}{l}\mathrm{Mn}-\mathrm{Co}- \\
\mathrm{P} / \mathrm{Ti}\end{array}$ & 76 & 135 & 52 & $\begin{array}{c}\text { Mn Doping of CoP Nanosheets } \\
\text { Array on Ti mesh }\end{array}$ & $\begin{array}{l}\text { ACS Catal. 2016, } \\
7(1), 98-102\end{array}$ \\
\hline $\mathrm{Fe}-\mathrm{CoP} / \mathrm{Ti}$ & 78 & & 75 & $\begin{array}{c}\text { Fe-doped CoP } \\
\text { nanoarray on Ti foil }\end{array}$ & $\begin{array}{l}\text { Adv. Mater. 2017, } \\
29(2), 1602441\end{array}$ \\
\hline $\mathrm{p}-\mathrm{CoP} / \mathrm{CP}$ & 57 & & & $\begin{array}{l}\text { Porous CoP nanosheets }(5 \sim 7 \mathrm{~nm}) \\
\text { on carbon paper }\end{array}$ & $\begin{array}{l}\text { Chem. Commun. 2018, } \\
54 \text { (12), 1465-1468 }\end{array}$ \\
\hline $\mathrm{CoPS} / \mathrm{CP}$ & 107 & 207 & 88.3 & $\begin{array}{c}\text { Cobalt phosphosulfide } \\
\text { nanoneedles in tetragonal phase } \\
\text { supported on carbon paper }\end{array}$ & $\begin{array}{c}\text { J. Mater. Chem. A 2018, } \\
\text { 6, 12353-12360 }\end{array}$ \\
\hline $\begin{array}{l}\text { Hollow } \\
\text { Mo-CoP }\end{array}$ & 40 & 130 & 65 & Hierarchical hollow Mo-CoP & Nano Energy 2018, \\
\hline
\end{tabular}




\begin{tabular}{|c|c|c|c|c|c|}
\hline nanoarrays & & & & nanoarrays on carbon cloth & $48,73-80$ \\
\hline $\begin{array}{l}\mathrm{CoP} @ \mathrm{NP} \\
\mathrm{MG}\end{array}$ & 150 & & 75 & $\begin{array}{l}\text { CoP nanoparticles anchored on } \\
\text { N,P-dual-doped graphene-like } \\
\text { carbon }\end{array}$ & $\begin{array}{l}\text { Nanoscale 2018, } \\
10(5), 2603-2612\end{array}$ \\
\hline $\begin{array}{l}\mathrm{NiCo}_{2} \mathrm{P}_{\mathrm{x}} / \mathrm{C} \\
\mathrm{F}\end{array}$ & 58 & & 34.3 & $\begin{array}{l}\text { Self-supported } \mathrm{NiCo}_{2} \mathrm{P}_{\mathrm{x}} \text { nanowire } \\
\text { arrays on commercial carbon felt }\end{array}$ & $\begin{array}{c}\text { Adv. Mater. 2017, } \\
29(9), 1605502\end{array}$ \\
\hline $\mathrm{CoP} \mathrm{NCs}$ & 62.5 & 150 & 69.2 & Colloidal CoP nanocrystals & $\begin{array}{c}\text { Adv. Mater. 2018, } \\
30(9), 1705796\end{array}$ \\
\hline $\mathrm{CoP} / \mathrm{CC}$ & 48 & 95 & 42.6 & $\begin{array}{c}\text { Rugae-like structure } \\
\text { on carbon cloth }\end{array}$ & $\begin{array}{c}\text { Nano Energy 2015, } \\
15,634-641\end{array}$ \\
\hline
\end{tabular}

* The value is obtained from the polarization curves shown in the literatures. 


\section{REFERENCES}

(1) Grahame, D. C. The Electrical Double Layer and the Theory of Electrocapillarity. Chem. Rev. 1947, $41(3), 441-501$.

(2) Conway, B.; Tilak, B. Interfacial Processes Involving Electrocatalytic Evolution and Oxidation of $\mathrm{H}_{2}$, and the Role of Chemisorbed H. Electrochim. Acta 2002, 47 (22-23), 3571-3594.

(3) Benck, J. D.; Chen, Z.; Kuritzky, L. Y.; Forman, A. J.; Jaramillo, T. F. Amorphous Molybdenum Sulfide Catalysts for Electrochemical Hydrogen Production: Insights into the Origin of Their Catalytic Activity. ACS Catal. 2012, 2 (9), 1916-1923.

(4) Chen, Z.; Cummins, D.; Reinecke, B. N.; Clark, E.; Sunkara, M. K.; Jaramillo, T. F. Core-Shell $\mathrm{MoO}_{3}-\mathrm{MoS}_{2}$ Nanowires for Hydrogen Evolution: A Functional Design for Electrocatalytic Materials. Nano Lett. 2011, 11 (10), 4168-4175.

(5) Popczun, E. J.; Read, C. G.; Roske, C. W.; Lewis, N. S.; Schaak, R. E. Highly Active Electrocatalysis of the Hydrogen Evolution Reaction by Cobalt Phosphide Nanoparticles. Angew. Chem. Int. Edit. 2014, $53(21), 5427-5430$.

(6) Kibsgaard, J.; Tsai, C.; Chan, K.; Benck, J. D.; Nørskov, J. K.; Abild-Pedersen, F.; Jaramillo, T. F. Designing an Improved Transition Metal Phosphide Catalyst for Hydrogen Evolution Using Experimental and Theoretical Trends. Energ. Environ. Sci. 2015, 8 (10), 3022-3029. 